\title{
Eficacia del zanamivir inhalado para el tratamiento y la prevención de la gripe
}

\author{
F. ABAD-SANTOS, S. GALLEGO SANDÍN, J. NOVALBOS REINA, E. MARTÍNEZ \\ SANCHO, M. A. GÁLVEZ MÚGICA
}

Servicio de Farmacología Clínica. Hospital Universitario de la Princesa. Madrid

\section{RESUMEN}

El objetivo de este trabajo es evaluar la eficacia del zanamivir en el tratamiento y la profilaxis de la gripe. Es un inhibidor de la neuraminidasa que es eficaz tanto contra el virus influenza A como B. Se han publicado 5 ensayos clínicos aleatorizados, controlados con placebo y con diseño doble-ciego, en los que reduce la duración de los síntomas de la gripe entre 1 y 2,5 días. El beneficio es mayor en los pacientes con infección confirmada por virus influenza, en los pacientes con fiebre y cuando el tratamiento se inicia en las primeras 30-36 horas. Para la profilaxis se han publicado dos ensayos clínicos controlados con placebo en los que reduce la incidencia de gripe un 50-67\%. Las características del zanamivir (eficacia, seguridad y falta de resistencias) le convierten en una opción adecuada para los sujetos no protegidos por la vacuna y para los que tienen alto riesgo de complicaciones.

PALABRAS CLAVE:Zanamivir. Inhibidores de neuraminidasa. Fármacos antivirales. Gripe. Revisión
EFFICACY OF INHALED ZANAMIVIR FOR THE TREATMENT AND PREVENTION OF INFLUENZA

\section{ABSTRACT}

The aim of this work is to evaluate the efficacy of zanamivir in the treatment and prevention of influenza. This drug is a neuraminidase inhi bitor that is effective against both $A$ and B influenza viruses. Five rando mized, double-blind, placebo-controlled, clinical trials have been publis hed, showing a reduction in the duration of flu symptoms between 1 and 2.5 days. A greater benefit is seen in the patients with influenza-confir med infection, with fever and when the treatment begins in the first 30-36 hours. For the prevention of influenza, two placebo-controlled clinical trials have been published, where flu incidence wass reduced by 50-67\%. The characteristics of zanamivir (efficacy, safety and lack of resistance) make it an appropriate option for subjects not protected by vaccination and for those with high risk of complications.

KEY WORDS: Zanamivir. Neuraminidase inhibitors. Antiviral agents. Influenza. Review

Abad-Santos F, Gallego Sandín S, Novalbos Reina J, Martínez Sancho E, Gálvez Múgica M.A. Eficacia del zanamivir inhalado para el tratamiento y la prevención de la gripe. An Med Interna (Madrid) 2001; 18: 93-99.

\section{INTRODUCCIÓN}

La gripe es una enfermedad muy frecuente que tiene una gran repercusión socioeconómica, ya que supone una causa importante de absentismo laboral. Además, existe un número importante de pacientes de alto riesgo, como son los ancianos y los que padecen enfermedades crónicas, en los que puede dar lugar a complicaciones como exacerbación aguda de bronquitis crónica, ataque agudo de asma, insuficiencia cardiaca congestiva, neumonía, otitis, sinusitis, etc., que en muchos casos conducen al ingreso hospitalario y a veces a la muerte del paciente (1). El agente causal es el virus influenza que se transmite rápidamente de unos individuos a otros con un periodo de incubación de 1 a 3 días, produciendo brotes epidémicos muy rápidos que duran unas 8-10 semanas (2).

A pesar de esto, todavía no se dispone de un tratamiento eficaz y seguro para esta enfermedad. Hasta ahora existían dos fármacos antivíricos (amantadina y rimantadina, aunque este último no está disponible en España), que están indicados en la profilaxis y el tratamiento de la gripe, pero su utilización está limitada por la alta incidencia de efectos adversos neurológicos (nerviosismo, insomnio, mareos, dificultad para concentrarse, psicosis), por el rápido desarrollo de resistencias víricas y porque no son eficaces contra el virus influenza B. Por estos motivos, la mejor medida terapéutica es la vacunación, aunque la eficacia varía según el grado de similitud entre la vacuna y las cepas causantes de la epidemia. 
El objetivo de este trabajo es evaluar la eficacia de un nuevo antivírico, el zanamivir, en la profilaxis y el tratamiento de la gripe, para lo que se han revisado todos los ensayos clínicos publicados con este fármaco. Con este propósito hemos realizado una búsqueda bibliográfica en MEDLINE en marzo de 2000 con la palabra zanamivir y más adelante se describen los ensayos clínicos encontrados.

\section{CARACTERÍSTICAS FARMACOLÓGICAS}

El zanamivir es un nuevo antivírico que inhibe específicamente la neuraminidasa del virus influenza, bloqueando de esta forma la replicación viral (3). La neuraminidasa es una enzima vírica que permite la liberación de viriones de las células infectadas, favorece la extensión de la infección por todo el tracto respiratorio y reduce la capacidad de la mucosa respiratoria para inactivar los virus. El zanamivir es un análogo del ácido siálico que se ha obtenido mediante estudios cristalográficos y diseño farmacológico racional asistido por ordenador (4). Como el sitio activo de la neuraminidasa es muy poco variable de unas cepas a otras, la probabilidad de que aparezca resistencia a zanamivir es muy baja (4), a diferencia de lo que ocurre con la amantadina y rimantadina que actúan sobre la proteína M2 que es más variable y no está presente en el virus influenza B. En estudios in vitro y en animales se ha comprobado que el zanamivir inhibe la propagación de los virus influenza A y B. No se ha comunicado ningún caso de resistencia clínicamente relevante del virus a este fármaco (3).

Como la absorción por vía oral es escasa y se elimina rápidamente por el riñón, se debe administrar por vía inhalatoria o intranasal, que además presenta la ventaja de actuar directamente allí donde se produce la replicación viral (3). Está comercializado en forma de inhalador de polvo seco (Diskhaler®) que con su uso correcto consigue que el 8-21\% del fármaco llegue a los pulmones; esta cantidad es suficiente para ejercer su efecto antivírico. El resto del fármaco se queda en la boca y en la faringe y posteriormente es deglutido (5).

\section{ENSAYOS CLÍNICOS EN EL TRATAMIENTO DE LA GRIPE}

El primer estudio para comprobar la eficacia del zanamivir en el tratamiento de la gripe se realizó en voluntarios sanos a los que se les contagió una gripe experimental inoculándoles por vía intranasal una cepa de virus influenza A (6). En un diseño aleatorizado, doble-ciego y controlado con placebo, se trataron con varias dosis de zanamivir por vía intranasal 2 o 6 veces al día durante 4 días, empezando 1 o 2 días después de la inoculación. El tratamiento administrado el primer día redujo los títulos virales en 2 logaritmos y la duración de la diseminación viral en 3 días y evitó la aparición de fiebre en un $85 \%$ de los sujetos. La eficacia del tratamiento iniciado en el segundo día fue ligeramente inferior. No se encontraron diferencias entre la administración 2 o 6 veces al día y no se detectaron efectos adversos graves. En los 57 sujetos inoculados con influenza A que recibieron tratamiento precoz en ese estudio, se comprobó que el zanamivir intranasal prevenía las alteraciones de la presión del oído medio (32\% con zanamivir frente a $73 \%$ con placebo), lo que puede suponer un marcador de que previene el riesgo de otitis media (7). Posteriormente se han publicado al menos 5 ensayos clínicos aleatorizados, doble-ciego y controlados con placebo, en los que se ha evaluado la eficacia del zana- mivir para el tratamiento de la gripe en la población general, y que vamos a comentar a continuación.

El primero de ellos se publicó en 1997 (8) y en realidad incluye dos ensayos clínicos realizados en 32 centros de Europa y 38 centros de Norteamérica en el invierno de 1994 a 1995. Participaron 417 pacientes previamente sanos que presentaban síntomas de gripe de menos de 48 horas de evolución, que se aleatorizaron a uno de tres grupos de tratamiento durante 5 días en un diseño doble ciego: zanamivir inhalado $10 \mathrm{mg} / 12 \mathrm{~h}$, zanamivir inhalado a la misma dosis más zanamivir intranasal $6,4 \mathrm{mg} / 12 \mathrm{~h}$, o placebo. Se excluyeron los pacientes que habían recibido la vacuna de la gripe. En el análisis por intención de tratar de todos los pacientes incluidos, el tiempo hasta el alivio de los síntomas principales (fiebre, cefalea, mialgia, tos e irritación de garganta) se redujo de 6 días en el grupo placebo a 5,3 días con zanamivir inhalado, independientemente de si habían recibido también zanamivir intranasal. Esta diferencia era estadísticamente significativa desde el tercer día de tratamiento.

Mediante técnicas de laboratorio que no están disponibles en la práctica clínica diaria, solamente se pudo confirmar el diagnóstico de infección por virus influenza en el $63 \%$ de los pacientes incluidos y los resultados fueron similares en este subgrupo (reducción de los síntomas de 0,9 días). No obstante, en los pacientes con infección por virus influenza que empezaron el tratamiento en las primeras 30 horas del comienzo de los síntomas, o que tenían fiebre mayor de $37,8^{\circ} \mathrm{C}$, los resultados fueron más marcados: la duración de los síntomas se redujo de 7 días con placebo a 4 días con zanamivir. En los pacientes sin fiebre o que iniciaron el tratamiento más de 30 horas después de empezar la sintomatología no se encontraron diferencias entre zanamivir y placebo.

En cuanto a otras variables, no se encontraron diferencias significativas en el consumo de paracetamol, ni en la incidencia de complicaciones que precisaron tratamiento antibiótico ( $8 \%$ con zanamivir y $12 \%$ con placebo). El zanamivir acortó el tiempo necesario para volver a las actividades normales en los pacientes que iniciaron el tratamiento en las primeras 30 horas: de 5,4 días con placebo a 4,1 días con zanamivir. El tratamiento fue bien tolerado y la mayoría de los eventos adversos estaban relacionados con la propia enfermedad.

El segundo ensayo clínico se publicó en 1999 (9) e incluyó 1256 pacientes mayores de 12 años, de Europa y Norteamérica, con síntomas gripales de menos de 48 horas de evolución durante una epidemia de gripe en el invierno de 1995 a 1996. Se asignaron aleatoriamente a recibir tratamiento con placebo o zanamivir $10 \mathrm{mg}$ inhalado más $6,4 \mathrm{mg}$ intranasal, 2 o 4 veces al día. En el 57\% de los pacientes se confirmó el diagnóstico de infección por virus influenza. En el análisis por intención de tratar, el zanamivir redujo significativamente la duración de los síntomas de 7 días con placebo a 6 días con zanamivir, sin encontrarse diferencias entre la administración en 2 o 4 veces al día. La reducción fue mayor en los pacientes que empezaron el tratamiento en las primeras 30 horas y en los pacientes con fiebre (acorta la duración entre 1 y 2 días). Además, el zanamivir disminuyó la intensidad de los síntomas, las alteraciones del sueño, la utilización de fármacos para el alivio de los síntomas y el tiempo hasta la reincorporación a las actividades normales (a los 5 días, el 45\% del grupo placebo estaban realizando sus actividades habituales, frente al $54-59 \%$ con zanamivir). 
En este estudio participaron 158 pacientes que tenían alto riesgo de desarrollar complicaciones de la gripe porque padecían enfermedades crónicas estables o porque eran mayores de 65 años. En este subgrupo de pacientes la reducción de la duración de los síntomas fue de 1,5 días con la pauta de 2 veces al día y de 2,75 días en los tratados 4 veces al día. En los pacientes de alto riesgo con confirmación de infección por virus influenza $(n=72)$, el zanamivir acortó la duración de los síntomas 2,75 días con la dosis baja y 3 días con la dosis alta.

Al igual que en el estudio anterior, el zanamivir fue bien tolerado a las dos dosis, siendo la incidencia de acontecimientos adversos y el número de pacientes que abandonó por eventos adversos igual que en el grupo placebo.

El tercer ensayo clínico se realizó en el invierno de 1997 en Australia, Nueva Zelanda y Sudáfrica y se publicó en 1998 (10). A través de una campaña de prensa se reclutaron 455 pacientes mayores de 12 años previamente sanos que presentaban síntomas gripales (fiebre o sensación febril asociada a dos o más de los siguientes síntomas: mialgia, tos, cefalea o irritación de garganta) de menos de 36 h de evolución. Los pacientes fueron aleatorizados a recibir tratamiento con placebo o zanamivir inhalado $10 \mathrm{mg}$ dos veces al día durante 5 días, y se les solicitó que anotasen en un diario la intensidad de los síntomas durante 14 días. La variable principal fue el tiempo hasta el alivio de los síntomas, que se redujo 1,5 días con zanamivir (5 días frente a 6,5 días con placebo). El 55\% de los pacientes presentaban fiebre al inicio del estudio y en ellos el zanamivir redujo la duración de los síntomas de 6,5 días a 4,5 días (intervalo de confianza del $95 \%$ de 1 a 4 días). En los pacientes sin fiebre documentada no se observó ningún efecto del tratamiento.

Se confirmó la infección por virus influenza en 321 pacientes $(71 \%)$, de los cuales $67 \%$ padecían influenza A y $33 \%$ influenza B. En los pacientes con gripe confirmada, el zanamivir redujo la duración de los síntomas de 6 días con placebo a 4,5 días, tanto en los pacientes con influenza $\mathrm{A}$ como con influenza B. En los pacientes en los que no se confirmó el diagnóstico de gripe, el zanamivir no fue eficaz. Además los pacientes tratados regresaron antes a sus actividades normales (7 días frente a 9 días con placebo), presentaron una menor puntuación de los síntomas, notificaron menos alteraciones del sueño y utilizaron menos antitusígenos, aunque estas dos últimas variables sólo fueron significativas en los pacientes con diagnóstico confirmado de influenza.

Sólo $76(17 \%)$ de los pacientes incluidos padecían otras enfermedades respiratorias, endocrinometabólicas o cardiovasculares, que se asocian a un mayor riesgo de complicaciones. En estos pacientes de alto riesgo también se observó una reducción de la duración de los síntomas de 8 días a 5,5 días. Además, en este subgrupo también se redujo de forma importante el número de pacientes que presentaron complicaciones (de $46 \%$ con placebo a $14 \%$ con zanamivir), y el número de pacientes que requirieron tratamiento antibiótico (de 36 a 14\%). Las complicaciones más frecuentes fueron bronquitis y neumonía.

El zanamivir fue bien tolerado, ya que la incidencia de acontecimientos adversos fue similar en los dos grupos de tratamiento: $37 \%$ de los pacientes que recibieron zanamivir y $43 \%$ del grupo placebo. Casi todos los eventos adversos fueron leves, siendo los más frecuentes tos, bronquitis, sinusitis, diarrea y náuseas o vómitos. Solamente interrumpieron el tratamiento por acontecimientos adversos 6 pacientes tratados con placebo y 4 con zanamivir.
El cuarto ensayo clínico se ha publicado en el año 2000 (11). Se realizó en Europa en el invierno de 1997 a 1998, con 356 pacientes que presentaban síntomas gripales de menos de 2 días. En un diseño doble-ciego se trataron con zanamivir inhalado $10 \mathrm{mg} / 12 \mathrm{~h}$ o placebo durante 5 días. El $78 \%$ de los pacientes presentaron un diagnóstico positivo para influenza, y en ellos el zanamivir produjo una reducción significativa de los síntomas: duración media de 7,5 días con placebo y de 5 días con zanamivir. Los resultados fueron similares en la población por intención de tratar. También disminuyó la intensidad de los síntomas, la utilización de paracetamol y dextrometorfano, y el número de complicaciones (de $34 \%$ con placebo a $23 \%$ con zanamivir). No obstante, solamente se incluyeron 32 pacientes de alto riesgo, por lo que no se pudo demostrar un beneficio significativo en este subgrupo de pacientes, aunque la tendencia era una reducción de la duración de los síntomas de 3,5 días, y las complicaciones se reducían de un $61 \%$ a un $33 \%$.

Los pacientes tratados con zanamivir se incorporaron antes al trabajo (7 días) que los que recibieron placebo $(8,5$ días). La incidencia de acontecimientos adversos fue inferior con zanamivir (25\% de los pacientes) que con placebo (35\%).

El quinto ensayo clínico se ha realizado en Japón (12) y ha incluido 116 pacientes con síntomas gripales de menos de 36 horas que fueron asignados a uno de 3 grupos de tratamiento durante 5 días: zanamivir inhalado $10 \mathrm{mg} / 12 \mathrm{~h}$, zanamivir inhalado a la misma dosis más zanamivir intranasal 6,4 mg/12 $\mathrm{h}$, o placebo. Los pacientes tratados con zanamivir se recuperaron más rápido (3 días) que los tratados con placebo (4 días). No se encontraron diferencias entre los dos grupos de zanamivir. El fármaco fue bien tolerado.

Además, se ha realizado otro ensayo clínico en Norteamérica en 777 pacientes, que todavía no se ha publicado pero sus resultados preliminares se han presentado en un congreso (3). En el se compara el zanamivir inhalado $10 \mathrm{mg}$ dos veces al día con placebo durante 5 días. La infección por virus influenza se confirmó en 511 pacientes, en los que el alivio de los síntomas se consiguió a los 6 días con placebo y a los 5 con zanamivir.

En conjunto, podemos ver que el zanamivir inhalado 10 mg dos veces al día durante 5 días acorta la duración de la gripe entre 0,7 y 2,5 días en todos los pacientes incluidos en los ensayos clínicos (Tabla I), entre 0,9 y 2,5 días en los pacientes con infección confirmada por virus influenza (Tabla II), entre 1,4 y 2 días en los pacientes con fiebre (Tabla III) y entre 1,5 y 3,5 días en los pacientes de alto riesgo (Tabla IV), aunque estos últimos datos no son estadísticamente significativos por el pequeño número de pacientes. En general, el beneficio es mayor en los pacientes con infección confirmada, en los pacientes con fiebre y cuando el tratamiento se inicia antes de 30-36 horas del inicio de los síntomas. Además, los pacientes tratados con zanamivir regresan a sus actividades normales 1,5 a 2 días antes que los que tomaron placebo.

Se ha realizado un análisis combinado de todos los pacientes que han recibido $10 \mathrm{mg} / 12 \mathrm{~h}$ de zanamivir inhalado $(\mathrm{n}=$ $1102)$ o placebo $(n=1133)$ durante 5 días en todos los ensayos clínicos realizados, con la intención de estimar el beneficio global del zanamivir en una población amplia y diversa (13). Se ha encontrado una reducción de un día en el tiempo hasta el alivio de los síntomas en toda la población y de 1,5 días en los pacientes con fiebre o con infección confirmada en el laboratorio. El beneficio era mayor en los pacientes con síntomas intensos (3 días) que en los pacientes con síntomas leves (1 día). Además, el zanamivir disminuía un $6 \%$ el consumo de paracetamol, un 


\section{TABLA I}

RESULTADOS EN LA POBLACIÓN POR INTENCIÓN DE

TRATAR EN LOS PRINCIPALES ENSAYOS CLÍNICOS CON ZANAM IVIR INHALADO 10 M G DOS VECES AL DÍA DURANTE 5 DÍAS EN EL TRATAM IENTO DE LA GRIPE

\begin{tabular}{|c|c|c|c|c|}
\hline \multirow[t]{2}{*}{ Estudio } & \multirow[t]{2}{*}{$n$} & \multicolumn{2}{|c|}{$\begin{array}{l}\text { Duración de los } \\
\text { síntomas (días** }\end{array}$} & \multirow{2}{*}{$\begin{array}{l}\text { Diferencia (días } \\
\text { (IC 95\%) }\end{array}$} \\
\hline & & placebo & zanamivir & \\
\hline Hayden et al (8) & 417 & 6,0 & 5,3 & $0,7(0$ a 1,4) \\
\hline Monto et al (9) & 1256 & 7,0 & $6,0 \$$ & $1,0(0$ a 2,0$)$ \\
\hline $\begin{array}{l}\text { The MIST study } \\
\text { group (10) }\end{array}$ & 455 & 6,5 & 5,0 & $1,5(0,5$ a 2,25$)$ \\
\hline M akela et al (11) & 356 & 7,5 & 5,0 & $2,5(0,75$ a 3,5 \\
\hline
\end{tabular}

* M ediana excepto en el estudio de Hayden et al, que son medias. \$Estos pacientes además recibían zanamivir intranasal.

\section{TABLA \|}

RESULTADOS EN LOS PACIENTES CON DIAGNÓ STICO CONFIRM ADO DE INFECCIÓN POR VIRUS INFLUENZA EN LOS PRINCIPALES ENSAYOS CLÍNICOS CON ZANAM IVIR INHALADO 10 M G DOS VECES AL DÍA DURANTE 5 DÍAS EN EL TRATAM IENTO DE LA GRIPE

\begin{tabular}{|c|c|c|c|c|}
\hline \multirow[t]{2}{*}{ Estudio } & \multirow[t]{2}{*}{$n$} & \multicolumn{2}{|c|}{$\begin{array}{l}\text { Duración de los } \\
\text { síntomas (días)* }\end{array}$} & \multirow{2}{*}{$\begin{array}{c}\text { Diferencia (días) } \\
\text { (IC 95\%) }\end{array}$} \\
\hline & & placebo & zanamivir & \\
\hline Hayden et al (8) & 262 & 6,3 & 5,4 & $0,9(0$ a 1,7$)$ \\
\hline M onto et al (9) & 722 & 7,0 & $5,5 \$$ & $1,5(-0,25$ a 2,0$)$ \\
\hline $\begin{array}{l}\text { The M IST study } \\
\text { group (10) }\end{array}$ & 321 & 6,0 & 4,5 & $1,5(0,5$ a 2,25$)$ \\
\hline Makela et al (11) & 277 & 7,5 & 5,0 & $2,5(1,0$ a 4,0$)$ \\
\hline
\end{tabular}

* M ediana excepto en el estudio de Hayden et al, que son medias. \$ Estos pacientes además recibían zanamivir intranasal.

TABLA III

RESULTADOS EN LOS PACIENTES CON FIEBRE EN LOS PRINCIPALES ENSAYOS CLÍNICOS CON ZANAM IVIR INHALADO $10 \mathrm{MG}$ DOS VECES AL DÍA DURANTE 5 DÍAS EN EL TRATAM IENTO DE LA GRIPE

\begin{tabular}{|c|c|c|c|c|}
\hline \multirow[t]{2}{*}{ Estudio } & \multirow[t]{2}{*}{$\mathrm{n}$} & \multicolumn{2}{|c|}{$\begin{array}{l}\text { Duración de los } \\
\text { síntomas (días)* }\end{array}$} & \multirow{2}{*}{$\begin{array}{l}\text { Diferencia (días) } \\
\qquad \text { (IC 95\%) }\end{array}$} \\
\hline & & placebo & zanamivir & \\
\hline Hayden et al (8) & 151 & 6,8 & 5,3 & $1,4(0,4$ a 2,5$)$ \\
\hline M onto et al (9) & 473 & 7,0 & $5,5 \$$ & $1,5(0$ a 2,5$)$ \\
\hline $\begin{array}{l}\text { The M IST study } \\
\text { group (10) }\end{array}$ & 251 & 6,5 & 4,5 & $2,0(1,0$ a 4,0$)$ \\
\hline
\end{tabular}

* M ediana excepto en el estudio de Hayden et al, que son medias. \$ Estos pacientes además recibían zanamivir intranasal.
TABLA IV

RESULTADOS EN LOS PACIENTES DE ALTO RIESGO EN LOS PRINCIPALES ENSAYO S CLÍNICOS CON ZANAM IVIR INHALADO 10 M G DOS VECES AL DÍA PARA EL TRATAM IENTO DE LA GRIPE

\begin{tabular}{|c|c|c|c|c|}
\hline \multirow[t]{2}{*}{ Estudio } & \multirow[t]{2}{*}{$n$} & \multicolumn{2}{|c|}{$\begin{array}{l}\text { Duración de los } \\
\text { síntomas (días)* }\end{array}$} & \multirow{2}{*}{$\begin{array}{l}\text { Diferencia (días } \\
\qquad \text { (IC 95\%) }\end{array}$} \\
\hline & & placebo & zanamivir & \\
\hline M onto et al (9) & 158 & 7,75 & $6,3 \$$ & $1,5(-0,5$ a 3,5$)$ \\
\hline $\begin{array}{l}\text { The M IST study } \\
\text { group (10) }\end{array}$ & 76 & 8,0 & 5,5 & $2,5(-1,0$ a 8,0$)$ \\
\hline M akela et al (11)\# & 32 & 11,5 & 8,0 & 3,5 (no significativ \\
\hline
\end{tabular}

* M ediana excepto en el estudio de Hayden et al, que son medias \$ Estos pacientes además recibían zanamivir intranasal.

\# En pacientes sin tratamiento sintomático.

$20 \%$ el consumo de antitusígenos y un 28\% la prescripción de antibióticos por complicaciones, y los pacientes regresaban a sus actividades un día antes. En un análisis de los 220 pacientes con influenza B se encontró una reducción de los síntomas de 1,5 días (3). En los pacientes de alto riesgo con diagnóstico confirmado de influenza $(\mathrm{n}=305)$ los síntomas se aliviaban 2,5 días antes, regresaban al trabajo 2 días antes y el consumo de antibióticos se reducía un 38\% (13).

\section{ENSAYOS CLIINICOS EN LA PREVENCIÓN DE LA GRIPE}

El primer estudio para comprobar la eficacia del zanamivir en la prevención de la gripe se realizó en 94 voluntarios sanos a los que se les contagió una gripe experimental inoculándoles por vía intranasal una cepa de virus influenza A (6). En un diseño aleatorizado, doble-ciego y controlado con placebo, se trataron con varias dosis de zanamivir por vía intranasal 2 o 6 veces al día durante 5 días, empezando 4 horas antes de la inoculación. El zanamivir previno en un $82 \%$ la aparición de infección por virus influenza confirmada en el laboratorio y en un $92 \%$ la aparición de enfermedad febril. En otro estudio similar con 34 voluntarios también demostró ser eficaz para prevenir la infección por influenza B (3). En un análisis conjunto de estos 128 sujetos inoculados con influenza A o B (7), la profilaxis por vía intranasal con zanamivir prevenía las alteraciones de la presión del oído medio ( $15 \%$ con zanamivir frente a $57 \%$ con placebo), lo que puede suponer un marcador de que previene el riesgo de otitis media.

En otro ensayo clínico de gripe experimental, doble-ciego, controlado con placebo (14), la administración de $600 \mathrm{mg}$ de zanamivir por vía intravenosa dos veces al día durante 5 días prevenía la diseminación viral ( $0 \%$ frente a $100 \%$ con placebo), disminuía la seroconversión ( $14 \%$ frente a $100 \%$ con placebo), la fiebre ( $14 \%$ frente a $88 \%$ ) y el resto de los síntomas. Ningún paciente tratado con zanamivir experimentó síntomas del tracto respiratorio superior frente a todos del grupo placebo.

El mayor estudio de profilaxis se publicó en 1999 (15). Se trata de un ensayo clínico aleatorizado, doble-ciego y controlado con placebo, en el que participaron 1107 adultos sanos durante el invierno de 1997 en dos comunidades universitarias del medio oeste americano. Los sujetos se aleatorizaron a recibir zanamivir $10 \mathrm{mg} /$ día inhalado o placebo durante 4 semanas a 
partir del inicio de la epidemia de gripe. No se incluyeron pacientes de alto riesgo. El zanamivir redujo la incidencia de gripe confirmada en el laboratorio de $6,1 \%$ en el grupo placebo a $2,0 \%$, lo que representa un riesgo relativo de 0,33 y una eficacia de un $67 \%$. También disminuyó la incidencia de gripe con fiebre de un $3,4 \%$ con placebo a un $0,5 \%$ con zanamivir, lo que supone una eficacia de un $84 \%$ para prevenir la gripe con fiebre (intervalo de confianza de 55 a 94\%). La incidencia de cualquier proceso febril se redujo de 10 a $6 \%$. El zanamivir fue bien tolerado y la incidencia de efectos adversos que el médico sospechaba que podían estar relacionados con el tratamiento fue similar en los dos grupos: $5,4 \%$ con zanamivir y $4,9 \%$ con placebo. Solamente $7(1,3 \%)$ pacientes del grupo placebo abandonaron el estudio por efectos adversos y $4(0,7 \%)$ del grupo de zanamivir.

En un ensayo clínico publicado este año (16) se evaluó la eficacia del zanamivir para prevenir la gripe en personas que han estado en estrecho contacto con pacientes diagnosticados de gripe, durante el invierno de 1995-1996 en Europa y Norteamérica. Se incluyeron 575 sujetos que se aleatorizaron de forma doble-ciego a uno de cuatro tratamientos durante 5 días: placebo, zanamivir inhalado $10 \mathrm{mg}$, zanamivir intranasal 6,4 mg, o zanamivir inhalado más intranasal una vez al día. Durante los 10 días posteriores al tratamiento desarrollaron gripe confirmada virológicamente el $8 \%$ del grupo placebo, el $6 \%$ de zanamivir intranasal, el 3\% de zanamivir inhalado y el $4 \%$ de zanamivir inhalado más intranasal. Aunque el zanamivir inhalado reducía más del $50 \%$ la incidencia de gripe no se alcanzó la significación estadística $(\mathrm{p}=0,066)$ por el pequeño número de sujetos contagiados.

En otro ensayo clínico se comparó el zanamivir $10 \mathrm{mg}$ inhalado y 3,2 mg intranasal dos veces al día con rimantadina $100 \mathrm{mg}$ /día por vía oral durante 14 días para la profilaxis de la influenza A en 65 y 23 pacientes, respectivamente, de una residencia de ancianos (17). Solo un paciente del grupo de rimantadina desarrolló gripe confirmada en el laboratorio, por lo que podemos suponer que la eficacia del zanamivir es igual o superior a rimantadina. En este mismo estudio, en una epidemia de influenza B se trataron 35 sujetos con zanamivir a la misma dosis y 18 sin tratamiento. Solamente un sujeto que no recibió tratamiento desarrolló una infección por influenza confirmada en el laboratorio, pero no se encontraron diferencias significativas por el pequeño tamaño de la muestra.

\section{SEGURIDAD}

Como hemos visto anteriormente, en todos los ensayos clínicos publicados, el zanamivir fue muy bien tolerado y no se encontró un aumento significativo de ningún efecto adverso con respecto a placebo. Los principales acontecimientos adversos comunicados fueron síntomas de vías respiratorias altas y síntomas digestivos que posiblemente se deban a la propia enfermedad. Tampoco produce alteraciones analíticas. No obstante, en Enero de 2000, la Agencia Española del Medicamento hizo pública una nota por la que recomendaba precaución en pacientes con asma o enfermedad pulmonar crónica, ya que existe riesgo de broncoespasmo, por lo que se deben administrar los broncodilatadores antes del tratamiento con zanamivir. Sin embargo, en un estudio reciente con 13 pacientes con asma leve-moderada que recibieron zanamivir inhalado cuatro veces al día durante 14 días, no se alteró significativamente la función pulmonar ni la reactividad de las vías aéreas (18).
En una revisión reciente se analiza la seguridad de zanamivir en los ensayos clínicos: casi 6.000 pacientes han sido incluidos en estos estudios, de los que 4.152 han sido tratados con este fármaco (19). La incidencia de efectos adversos es similar al placebo y no se han detectado alteraciones analíticas. En algunos estudios en voluntarios sanos, la administración de dosis de hasta $600 \mathrm{mg}$ por vía intravenosa ha sido bien tolerada. Como la absorción sistémica es muy baja y no es metabolizado, no se prevé que se produzcan interacciones con otros fármacos.

El zanamivir no ha sido autorizado para niños menores de 12 años ni para mujeres embarazadas o en periodo de lactancia por falta de experiencia en estos grupos (5).

\section{DISCUSIÓN}

Podemos concluir que el zanamivir es un fármaco eficaz y seguro para la prevención y el tratamiento de la gripe, tanto de influenza A como B. En el caso del tratamiento es recomendable iniciarlo en las primeras 30-36 horas de aparición de los síntomas y consigue acortar la duración de los mismos en 1 a 2,5 días. En la profilaxis existen menos ensayos clínicos, pero parece reducir la incidencia de gripe a un tercio.

Es posible que alguien piense que el beneficio es pequeño, pero para un paciente esto puede ser clínicamente relevante, especialmente si se consigue con un tratamiento exento de efectos adversos. De todos modos, ésta es la media de mejoría, por lo que unos pacientes se beneficiarán más y otros menos. De hecho en los pacientes con confirmación de infección por virus influenza que son tratados más precozmente y que tienen fiebre, el beneficio es superior (de hasta 3 días). Desde el punto de vista sociosanitario es especialmente relevante que los pacientes vuelvan a sus actividades normales 2 días antes y que disminuyan las complicaciones de la gripe. Además, puede reducir la mortalidad y mejorar la calidad de vida de los pacientes. Si se confirma que el zanamivir reduce la duración de las bajas laborales y disminuye los ingresos hospitalarios y la mortalidad, presentará una relación costebeneficio muy favorable. No obstante, es necesario realizar estudios farmacoeconómicos para demostrarlo.

El zanamivir supone un avance terapéutico importante en la lucha contra la gripe (20), aunque todavía son necesarios más ensayos clínicos en los pacientes de alto riesgo. Hasta ahora los únicos fármacos aprobados para la profilaxis y el tratamiento de la gripe eran la amantadina y la rimantadina. Para la profilaxis de la influenza A la amantadina y la rimantadina son tan eficaces como el zanamivir, previenen el 70$90 \%$ de los casos (21), pero tienen los inconvenientes de producir muchos efectos adversos, dar lugar a la aparición de resistencias y no ser eficaces frente a la influenza B.

Existe otro fármaco inhibidor de la neuraminidasa, el oseltamivir, que va a ser comercializado próximamente y que se diferencia porque se administra por vía oral, lo cual puede ser más cómodo para algunos pacientes. También se diferencian porque actúan en distinto sitio de la enzima. En varios estudios aleatorizados y controlados con placebo, el oseltamivir ha demostrado que produce una eficacia similar al zanamivir para la profilaxis (22) y el tratamiento de la gripe (23). No obstante, el oseltamivir suele producir náuseas en el 12-15\% de los pacientes en los estudios de profilaxis (22) y en el $17-19 \%$ en los estudios de tratamiento porque se administra una dosis más alta (23), frente a sólo un $7 \%$ con placebo. También produce vómitos con una 
incidencia de $2-3 \%$ en profilaxis $(0,8 \%$ con placebo) y $13-15 \%$ en tratamiento $(3 \%$ con placebo) $(22,23)$. Por lo tanto, la comodidad de la vía de administración de oseltamivir se ve contrariada por la incomodidad de los efectos adversos gastrointestinales, con lo cual la principal ventaja del zanamivir reside en su buena tolerabilidad debido a la escasa absorción sistémica.

La vacunación es actualmente la medida más efectiva para reducir el impacto de las epidemias de gripe. No obstante, es difícil preparar una vacuna para un virus altamente variable y con cambios continuos (24). Otras desventajas de la vacuna son que no está exenta de efectos adversos y que es necesario administrarla todos los años. La eficacia de la vacuna en un año concreto y para un paciente determinado puede ser muy variable porque depende de los siguientes factores (21):

1. Factores relacionados con la vacuna, como la preparación, la dosis y el grado de relación antigénica con las cepas circulantes.

2. Factores virológicos y epidemiológicos, que incluyen el intervalo entre la vacunación y la exposición, y la virulencia y el grado de transmisibilidad de las cepas epidémicas.

3. Factores del huésped como la edad, el estado de salud y la experiencia antigénica previa.

La efectividad de la vacuna depende ampliamente de la similitud antigénica entre los virus que están produciendo una epidemia y los virus contenidos en la vacuna administrada. En los últimos años, esta similitud es alta gracias a los esfuerzos continuos de vigilancia epidemiológica realizados por un grupo de laboratorios internacionales coordinados por la Organización Mundial de la Salud. Incluso en condiciones ideales en las que hay una gran similitud entre la vacuna y las cepas circulantes, la efectividad de la vacuna se encuentra en el rango de 70 a $90 \%$ para adultos jóvenes sanos, pero puede disminuir sustancialmente en pacientes ancianos, con una efectividad de sólo $30-50 \%$ (25). Actualmente se recomienda la vacunación de todos los pacientes con alto riesgo de complicaciones (ancianos y pacientes con enfermedades crónicas) y del personal sanitario o cuidadores que pueden transmitir la enfermedad a personas de alto riesgo. La variabilidad de la eficacia protectora y la dificultad para conseguir que se vacunen todos los pacientes de alto riesgo, deja un gran número de pacientes no inmunizados que podrían ser susceptibles de otras medidas terapéuticas (21). Además, no se vacuna a las personas sanas, con lo cual en ellas podría ser muy útil la administración de una profilaxis eficaz cuando tienen un alto riesgo de ser contagiadas, o de un tratamiento adecuado cuando contraen la enfermedad.

El zanamivir no debe sustituir a la vacunación anual para la gripe, sino que puede ser útil para tratar a aquellos pacientes que no se han vacunado o en los que la vacuna está contraindicada. El zanamivir no altera la respuesta a la vacuna de la gripe (26) por lo que se podría utilizar para proteger a los pacientes de riesgo en las 2-4 semanas que tarda en desarrollarse la inmunidad. La extensión de las epidemias de gripe es muy rápida por lo que el tiempo para reaccionar desde que se reconoce un cambio en un nuevo subtipo del virus hasta que empieza una pandemia puede ser demasiado corto para preparar la vacuna, administrarla y desarrollar inmunidad en los pacientes de alto riesgo.

El diagnóstico clínico de la gripe es poco específico; de hecho, en los ensayos clínicos solamente en el 58 a $73 \%$ de los pacientes incluidos se confirmó la infección por virus influenza. Para poder aplicar estos tratamientos solamente en aquellos pacientes con gripe se necesitan pruebas diagnósticas rápidas que permitan diferenciar en la consulta del médico entre una infección por virus influenza y otras infecciones por otros virus. En Estados Unidos ya se han comercializado varios de estos tests que proporcionan un diagnóstico rápido, en menos de $30 \mathrm{~min}$, con una sensibilidad que, dependiendo del test, oscila entre 60 y $81 \%$, y una especificidad superior al $93 \%$ (27). Como la sensibilidad no es muy alta, un resultado negativo no excluye la infección por virus influenza. El problema es que estas técnicas serán caras y todavía no son accesibles para todos los pacientes. Posiblemente cuando una epidemia de gripe está bien caracterizada, el diagnóstico clínico sea suficiente para guiar el tratamiento.

El zanamivir ha sido autorizado para el tratamiento de la gripe en la mayor parte de los países desarrollados y en algunos también para la profilaxis, pero en la mayoría de ellos no es financiado por las autoridades sanitarias, como ocurre en España, porque consideran que su eficacia es limitada. Por este motivo, es el paciente quien debe decidir si vale la pena pagarse esta medicación para acortar la duración de los síntomas en 1-2,5 días. El principal inconveniente postulado por las agencias reguladoras para no financiarlo es que no se ha demostrado de forma contundente que disminuya las complicaciones o la necesidad de hospitalización o aumente la supervivencia en pacientes de alto riesgo (5). No obstante, con los datos disponibles hasta ahora parece claro que previene las complicaciones y que la eficacia en los pacientes de alto riesgo es mayor que en la población general (reducción de los síntomas entre 1,5 y 3,5 días), aunque se siguen realizando ensayos clínicos en este grupo de pacientes.

Otro aspecto criticado es la limitada eficacia (0,7-2,5 días) (28), pero si nos fijamos en el intervalo de confianza para algunos subgrupos, como los pacientes con fiebre tratados antes de $30 \mathrm{~h}$, la reducción puede llegar a ser de hasta 4 días, lo cual parece muy relevante para una enfermedad que dura alrededor de 7 días. Es el paciente el que debería decidir si este beneficio es suficiente para mejorar su calidad de vida. Debemos tener en cuenta que el zanamivir es muy bien tolerado, solo hay que tomarlo 5 días y para un paciente concreto le puede suponer que pierda 1,2,3 ó 4 días menos de desarrollar su actividad normal y de sufrir una sintomatología molesta. Como el tratamiento es más eficaz cuanto antes empiece a administrarse, podríamos pensar que la autoprescripción sería adecuada, pero como existe un alto riesgo de errores diagnósticos la prescripción debería ser realizada por un facultativo.

\section{CONCLUSIÓN}

El zanamivir ha demostrado claramente su eficacia para el tratamiento de la gripe, aunque en los ensayos clínicos realizados se han incluido pocos pacientes de alto riesgo por lo que se siguen haciendo estudios en este subgrupo de población. Para la profilaxis existe un menor número de ensayos pero los resultados también son muy favorables.

Aunque la vacunación de poblaciones seleccionadas sigue siendo la medida de primera elección para la profilaxis de la gripe, las características del zanamivir (eficacia, seguridad y falta de resistencias) le convierten en una opción adecuada para los sujetos no protegidos por la vacuna y para los que tienen alto riesgo de complicaciones. 


\section{Bibliografía}

1. Curwen M, Dunnell K, Ashley J. Hidden influenza deaths. BMJ 1990; 300: 1996.

2. Fleming DM, Zambon M, Bartelds AIM, de Jong JC. The duration and magnitude of influenza epidemics: a study of surveillance data from sentinel general practices in England, Wales and the Netherlands. Eur $\mathbf{J}$ Epidemiol 1999; 15: 467-473.

3. Dunn CJ, Goa KL. Zanamivir: a review of its use in influenza. Drugs 1999; 58: 761-784.

4. Von Itzstein M, Wu W-Y, Kok GB, et al. Rational design of potent sialidasebased inhibitors of influenza virus replication. Nature 1993; 363: 418-423.

5. Anónimo. Zanamivir for influenza. Drug Ther Bull 1999; 37: 81-84.

6. Hayden FG, Treanor JJ, Betts RF, Lobo M, Esinhart JD, Hussey EK. Safety and efficacy of the neuraminidase inhibitor GG167 in experimental human influenza. JAMA 1996; 275: 295-299.

7. Walker JB, Hussey EK, Treanor JJ, Montalvo A Jr, Hayden FG. Effect of the neuraminidase inhibitor zanamivir on otologic manifestations of experimental human influenza. J Infect Dis 1997; 176: 14717-1422.

8. Hayden FG, Osterhaus ADME, Treanor JJ, et al. Efficacy and safety of the neuraminidase inhibitor zanamivir in the treatment of influenzavirus infections. N Engl J Med 1997; 337: 874-880.

9. Monto AS, Fleming DM, Henry D, et al. Efficacy and safety of the neuraminidase inhibitor zanamivir in the treatment of influenza A and B virus infection. J Infect Dis 1999; 180: 254-261.

10. The MIST Study Group. Randomised trial of efficacy and safety of inhaled zanamivir in treatment of influenza A and B virus infections. Lancet 1998; 352: 1877-1881.

11. Makela MJ, Pauksens K, Rostila T, et al. Clinical efficacy of the orally inhaled neuraminidase inhibitor zanamivir in the treatment of influenza: a randomized, double-blind, placebo-controlled European study. J Infect 2000; 40: 42-48.

12. Matsumoto K, Ogawa N, Nerome K, et al. Safety and efficacy of the neuraminidase inhibitor zanamivir in treating influenza virus infection in adults: results from Japan. GG167 Group. Antivir Ther 1999; 4: 61-68.

13. Monto AS, Webster A, Keene O. Randomized, placebo-controlled studies of inhaled zanamivir in the treatment of influenza A and B: pooled efficacy analysis. J Antimicrob Chemother 1999; 44 (supl. B): 23-29.

14. Calfee DP, Peng AW, Cass LM, Lobo M, Hayden FG. Safety and efficacy of intravenous zanamivir in preventing experimental human influenza A virus infection. Antimicrob Agents Chemother 1999; 43: 1616-1620.
15. Monto AS, Robinson DP, Herlocher ML, Hinson JM Jr, Elliot MJ, Crisp A. Zanamivir in the prevention of influenza among healthy adults: a randomized controlled trial. JAMA 1999; 282: 31-35.

16. Kaiser L, Henry D, Flack NP, Keene O, Hayden FG. Short-term treatment with zanamivir to prevent influenza: results of a placebo-controlled study. Clin Infect Dis 2000; 30: 587-589.

17. Schilling M, Povinelli L, Krause P, et al. Efficacy of zanamivir for chemoprophylaxis of nursing home influenza outbreaks. Vaccine 1998; 16: 1771-1774.

18. Cass LM, Gunawardena KA, Macmahon MM, Bye A. Pulmonary function and airway responsiveness in mild to moderate asthmatics given repeated inhaled doses of zanamivir. Respir Med 2000; 94: 166173.

19. Freund B, Gravenstein S, Elliot M, Miller I. Zanamivir: a review of clinical safety. Drug Saf 1999; 21: 267-281.

20. Gubareva LV, Kaiser L, Hayden FG. Influenza virus neuraminidase inhibitors. Lancet 2000; 355: 827-835.

21. Patriarca PA. New options for prevention and control of influenza. JAMA 1999; 282: 75-77.

22. Hayden FG, Atmar RL, Schilling M, et al. Use of the selective oral neut raminidase inhibitor oseltamivir to prevent influenza. N Engl J Med 1999; 341: 1336-1343.

23. Treanor JT, Hayden FG, Vrooman PS, et al. Efficacy and safety of the oral neuraminidase inhibitor oseltamivir in treating acute influenza: a randomized controlled trial. JAMA 2000; 283: 1016-1024.

24. Cox NJ, Hughes JM. New options for the prevention of influenza. N Engl J Med 1999; 341: 1387-1388.

25. Gross PA, Hermogenes AW, Sacks HS, Lau J, Levandowski RA. The efficacy of influenza vaccine in elderly persons. Ann Med Intern 1995; 123: 518-527.

26. Webster A, Boyce M, Edmundson S, Miller I. Coadministration of orally inhaled zanamivir with inactivated trivalent influenza vaccine does not adversely affect the production of antihaemagglutinin antibodies in the serum of healthy volunteers. Clin Pharmacokinet 1999; 36 (supl. 1): 51-58.

27. Anónimo. Pruebas rápidas de diagnóstico de la gripe. Med Lett Drug Ther (ed española) 2000; 22: 13 .

28. Anónimo. Zanamivir: no tangible progress against influenza. Prescrire International 2000; 9: 199-202 